\title{
Antibiotic regimens utilization in treating community acquired pneumonia of a government practice setting: a prospective observational study in medical inpatients
}

\author{
K. Leela Prasad Babu1, Shaik Kareemulla ${ }^{2 *}$, S. K. Sai Pravalika², P. Nishad Khan², \\ Govindu Venkateswarlu ${ }^{2}$, K. Sreeharinadh ${ }^{2}$
}

\begin{abstract}
${ }^{1}$ Department of General Medicine, Rajiv Gandhi Institute of Medical Sciences, Kadapa, Andhra Pradesh, India
${ }^{2}$ Department of Pharmacy Practice, P. Rami Reddy Memorial College of Pharmacy, Kadapa, Andhra Pradesh, India
\end{abstract}

Received: 30 September 2019

Revised: 07 December 2019

Accepted: 09 December 2019

\section{*Correspondence:}

Dr. Shaik Kareemulla,

Email: tanveerkareems@gmail.com

Copyright: (C) the author(s), publisher and licensee Medip Academy. This is an open-access article distributed under the terms of the Creative Commons Attribution Non-Commercial License, which permits unrestricted non-commercial use, distribution, and reproduction in any medium, provided the original work is properly cited.

\begin{abstract}
Background: Pneumonia is a lower respiratory tract infection characterized by inflammation of lung tissue accompanied by infiltration of alveoli and bronchioles. Most common type is community acquired pneumonia (CAP). Initial therapy is usually empirical that is designed to treat various pathogens. In CAP cases, antibiotic therapy should begin at the earliest. The objectives of the study include to identify most common causative micro-organisms, to assess risk of developing CAP in patients having co-morbidities, to identify most commonly prescribed antibiotic regimen.

Methods: A prospective observational study was conducted for period of 6 months at RIMS, Kadapa. 120 patients were recruited based on inclusion criteria. Treatment was given according to Infectious Diseases Society of America and American Thoracic Society guidelines.

Results: In a total of 120 patients, 77 were males and 43 were females. 69 patients belong to $46-55 \&$ above age groups. 84 patients had social habits and 36 patients are without social habits. Patients with single lobe infiltrations are 105 and patients with multiple lobe infiltrations are 15. In our study, streptococcus pneumoniae and pseudomonas aeruginosa were the most common isolated organisms. Monotherapy was given for 7 patients, dual therapy for 97 patients and triple therapy for 16 patients. 33 patients received ceftriaxone (CEF) and augmentin (AUG), 29 patients received CEF and azithromycin (AZI), 7 patients received levofloxacin (LEV), 25 patients received CEF and LEV, 10 patients received CEF and ciprofloxacin (CIP) and 16 patients received CEF, AUG and AZI. 100 patients had less than 8 days of hospital stay.

Conclusions: Research study concluded that $\beta$ lactum antibiotics were the most commonly prescribed class. CEF and AUG was highly recommended drug regimen.
\end{abstract}

Keywords: Streptococcus pneumonia, Empirical therapy, Inflammation, Bronchioles

\section{INTRODUCTION}

Pneumonia is a lower respiratory tract infection characterized by collection of pus and other fluids in the air sacs i.e., alveoli of the lungs. Lung air sacs are structures, play a significant role in the exchange of gases such as oxygen and carbon dioxide. Collection of pus in air sacs leads to shortness of breath i.e., dyspnea. Pneumonia is caused by bacteria such as Streptococcus pneumoniae, Mycoplasma pneumoniae, 
Clamidophila pneumoniae, fungus such as Legionella species and other respiratory viruses. ${ }^{1}$ Pneumonia is an infection of lung parenchyma classified into community acquired pneumonia (CAP) and health care associated pneumonia (HCAP). ${ }^{2}$ The increased incidence of CAP with increasing patient age; the annual incidence of pneumonia in the USA was 24.8 cases per 10,000 adults with the highest rates among adults aged, between 65 and 79 years of age (63.0 cases per 10,000 adults) and those aged 80 years or older ( 164.3 cases per 10,000 adults). ${ }^{3}$ The most common symptoms include cough that produces sputum, fever, shortness of breath, headache. ${ }^{4}$ Classic pneumonia is caused due to Streptococcus pneumonia, in this, lobar patterns are present, evolves through four phases and is characterized by changes in the alveoli: stage of congestion, stage of red hepatization, stage of grey hepatization, stage of resolution..$^{5}$ For pneumonia diagnosis patients detail history of sign and symptoms, chest X-rays, sputum test, culture sensitivity test are useful. ${ }^{6}$ According to Infectious Disease Society of America and American Thoracic Society, Initial therapy is usually empirical that is designed to treat CAP caused due to various pathogens. In CAP cases, antibiotic therapy should begin at the earliest. ${ }^{7}$ To study the empirical treatment of antibiotic drug regimens in patients with CAP in a tertiary care teaching hospital. The main objectives of our study were to identify the most common causative micro-organisms to assess the risk of developing community acquired pneumonia in patients having co-morbidities, to assess the empirical antibiotic treatment, to identify the most commonly prescribed class of antibiotics for treating CAP.

\section{METHODS}

A hospital based prospective observational study was carried out in general medicine identified patients (IP) departments at Rajiv Gandhi Institute of Medical Sciences (RIMS), Kadapa for a period of 6 months i.e., from June 2018 to December 2018. RIMS Hospital is a 750 bedded multi-disciplinary tertiary care teaching hospital. A total of 120 patients prescribed with different antibiotics such as levofloxacin (LEV), ceftriaxone (CEF) and augmentin (AUG), CEF and azithromycin (AZI), CEF and LEV, CEF and ciprofloxacin (CIP), CEF, AUG and AZI. Patient who are willing to participate in the study. Both male and female patients (IP patients) who were diagnosed as CAP and prescribed with antibiotic drug regimen. Patients with co-morbid pathological conditions also included in our study. Patients who are not willing to participate in the study, Neonates and pediatric patients are excluded. Pregnant women, out patients and patients admitted for surgical procedures also excluded. Ethical approval was taken from respective ethical committee. After taking consent from the patient, a self-designed patient data collection form was used to collect data such as demographic details, provisional diagnosis, confirmatory diagnosis, radiographic data, social habitats, isolated organism, sensitivity and resistance to various antibiotics, treatment before culture report and after culture report of specimens of patients with CAP during hospital stay. Complete information was collected, analyzed and documented. Patients were also provided with patient information leaflets at the time of discharge.

\section{Statistical analysis}

Results were represented as frequencies, percentages, mean and medians. Percentage method was used for analyzing the data. Graph pad prism software was applied to analyze the data. In some cases, inferential statistics like analysis of variance (ANOVA) followed by student t-test, (at 95\% confidence interval and $\mathrm{p}<0.05$ considered as significant) using SPSS 21.0 software.

\section{Ethical considerations}

One of the major characteristics of modern society is a pronounced interest in ethical questions. Medicine, especially research on humans, is at the top of the list. The most important ethical aspect of the clinical study is informed consent document. The international covenant accepted by United Nations Assembly in 1966 stresses that "no one shall be subjected without his free consent to medical or scientific experimentation".

\section{RESULTS}

\section{Patient distribution based on gender}

Out of 120 patients, 77 were males constituted for $64.17 \%$ and 43 were females constituted for $35.83 \%$. In our analysis, we found that community acquired pneumonia is more common in males when compared to females. This states that males are at higher risk of developing community acquired pneumonia because majority of male patients were prone to have social habits.

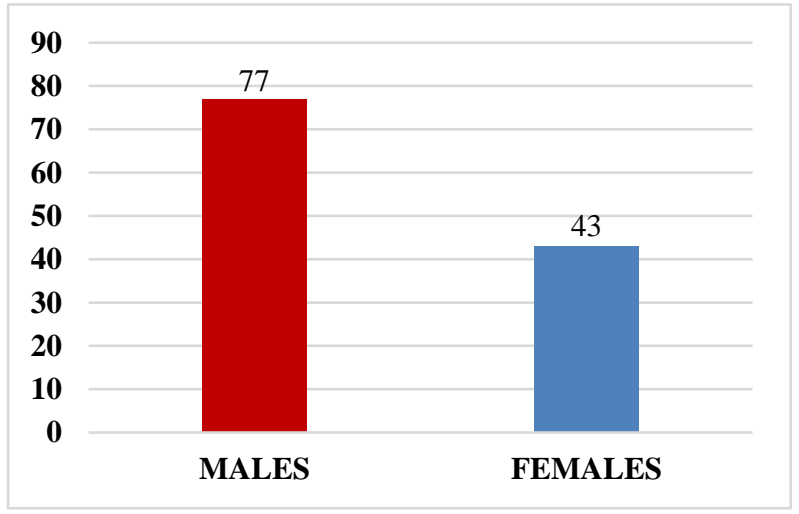

Figure 1: Gender distribution.

\section{Patient distribution based on different age groups}

A total of 120 patient case sheets diagnosed with CAP were collected and divided into 5 different age groups. 12 patients were in 16-25 years age group constituted for 
$10 \%, 16$ patients were in 26-35 years age group constituted for $13.33 \%, 23$ patients were in 36-45 years age group constituted for $19.17 \%, 38$ patients were in 4655 years age group constituted for $31.67 \%$ and 31 patients were in 56 and above years age group constituted for $25.83 \%$.

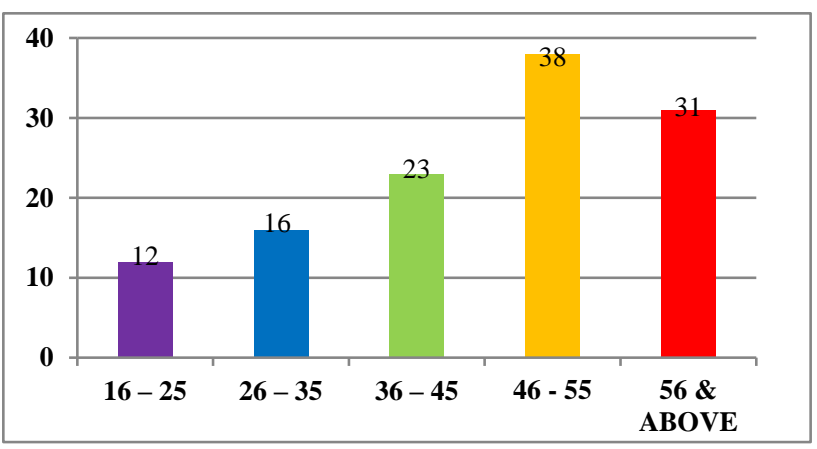

Figure 2: Different age groups patients.

\section{Patient distributions based on detailed types of co- morbidities}

In a total of 120 patients, 58 patients had co-morbid conditions of which 17 patients had co-morbidities with chronic obstructive pulmonary disease (COPD) constituted for $29.31 \%$, 6 patients had co-morbidities with asthma constituted for $10.34 \%, 12$ patients had co-morbidities with hypertension constituted for $20.69 \%$, 14 patients had co-morbidities with type 2 diabetes mellitus (T2DM) constituted for $24.14 \%, 4$ patients had co-morbidities with hypothyroidism constituted for $6.90 \%, 2$ patients had co-morbidities with epilepsy constituted for $3.45 \%, 3$ patients had co-morbidities with ischemic heart disease (IHD) constituted for $5.17 \%$.

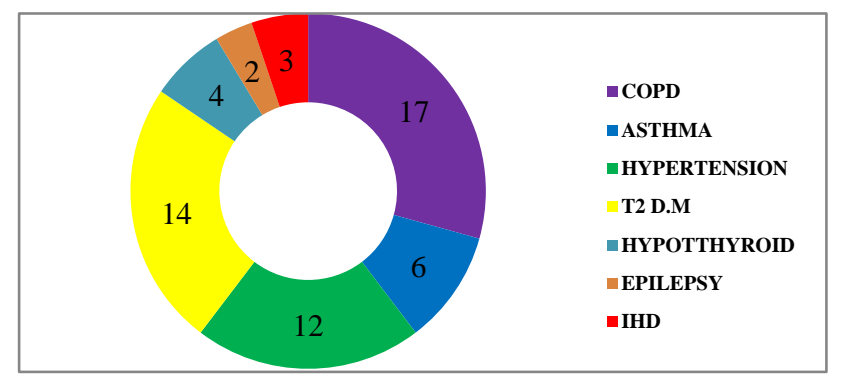

Figure 3: Patients with detailed co-morbidities.

\section{Patient distribution based on detailed social habits}

A total of 120 subjects were selected for our research study in which 84 patients had social habits and 36 patients did not have any of social habits. Out of 84 subjects, 35 patients had a habit of Smoking constituted for $29.16 \%, 12$ patients were Alcoholics constituted for $10.00 \%, 17$ patients were beetle nut chewers constituted for $14.16 \%$ and 20 patients had a habit of both smoking and alcoholism constituted for $16.66 \%$.

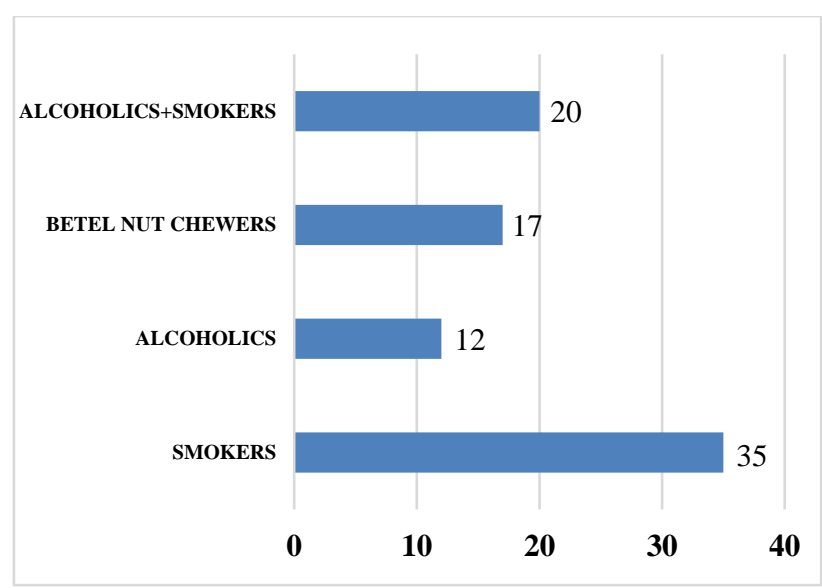

Figure 4: Patients with detailed social habits.

\section{Patient distribution based on radiological findings}

In our research study, 120 patients who were recruited according to inclusion criteria have undergone radiological examinations such as chest X-ray, CT chest and found that 105 patients had single-lobe infiltration constituted for $87.50 \%$ and remaining 15 patients had multi-lobe infiltration constituted for $12.50 \%$.

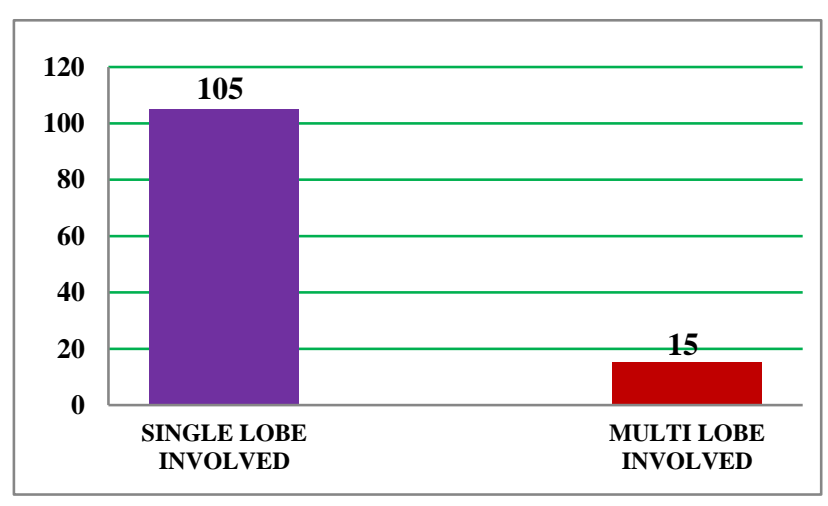

Figure 5: Single-lobe and multi-lobe infiltrations.

\section{Patients distribution based on sputum culture test}

In a total of 120 patients, 65 patients had undergone sputum culture test constituted for $54.17 \%$ and remaining 55 patients did not undergo sputum culture tests constituted for $45.83 \%$.

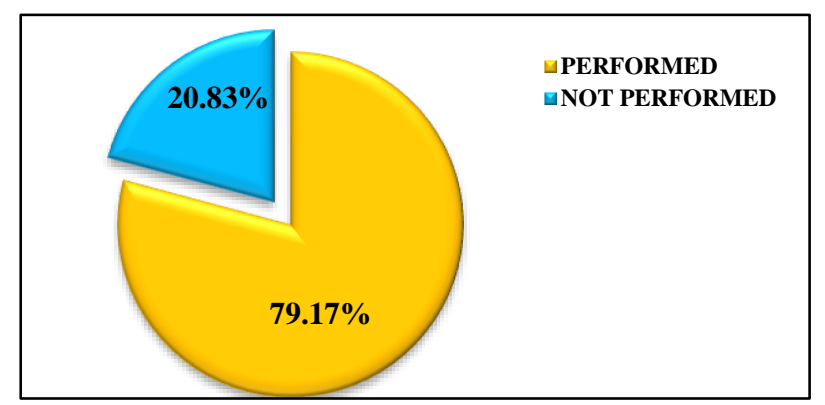

Figure 6: Patients with respect to sputum culture test. 


\section{Patient distribution based on types of bacteria}

In a total of 68 samples, it was found that Streptococcus pneumoniae was isolated in 23 patients constituted for $33.82 \%$, Pseudomonas aeruginosa was isolated in 19 patients constituted for $27.94 \%$, Klebsiella pneumoniae was isolated in 11 patients constituted for $16.18 \%$ Escherichia coli was isolated in 7 patients constituted for $10.29 \%$, Streptococcus aureus was isolated in 6 patients constituted for $8.82 \%$ and Hemophilus influenzae was isolated in 2 patients constituted for $2.95 \%$.

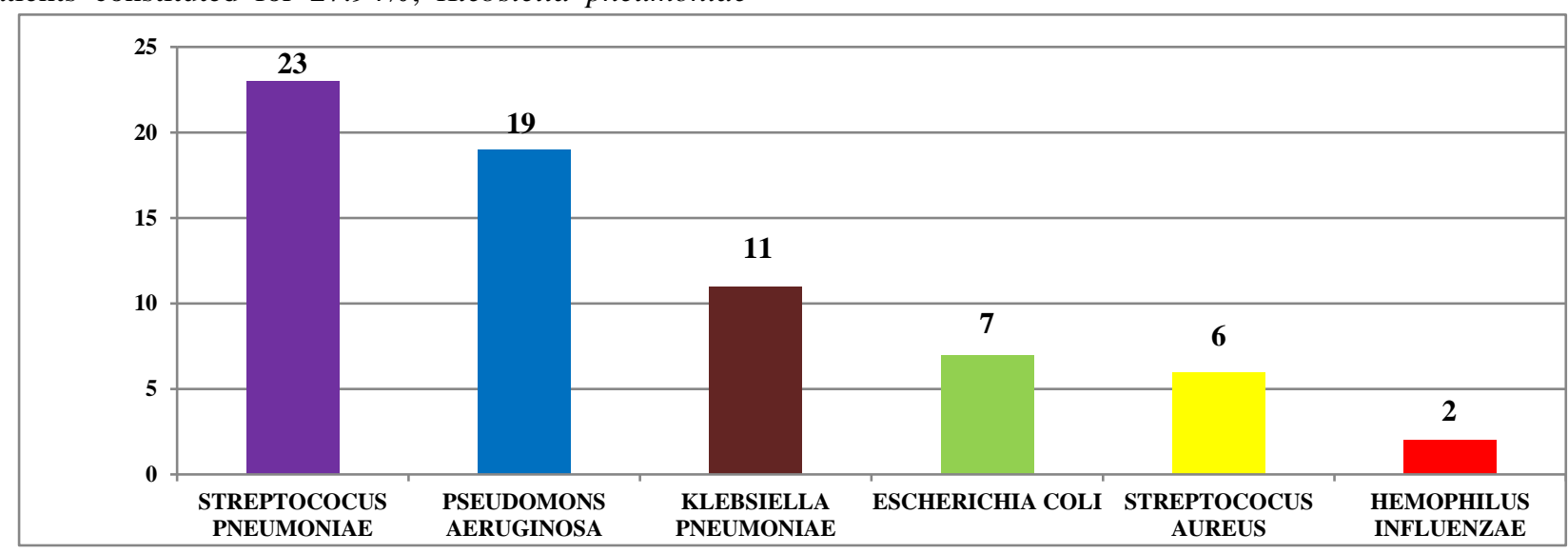

Figure 7: Patients based on types of bacteria.

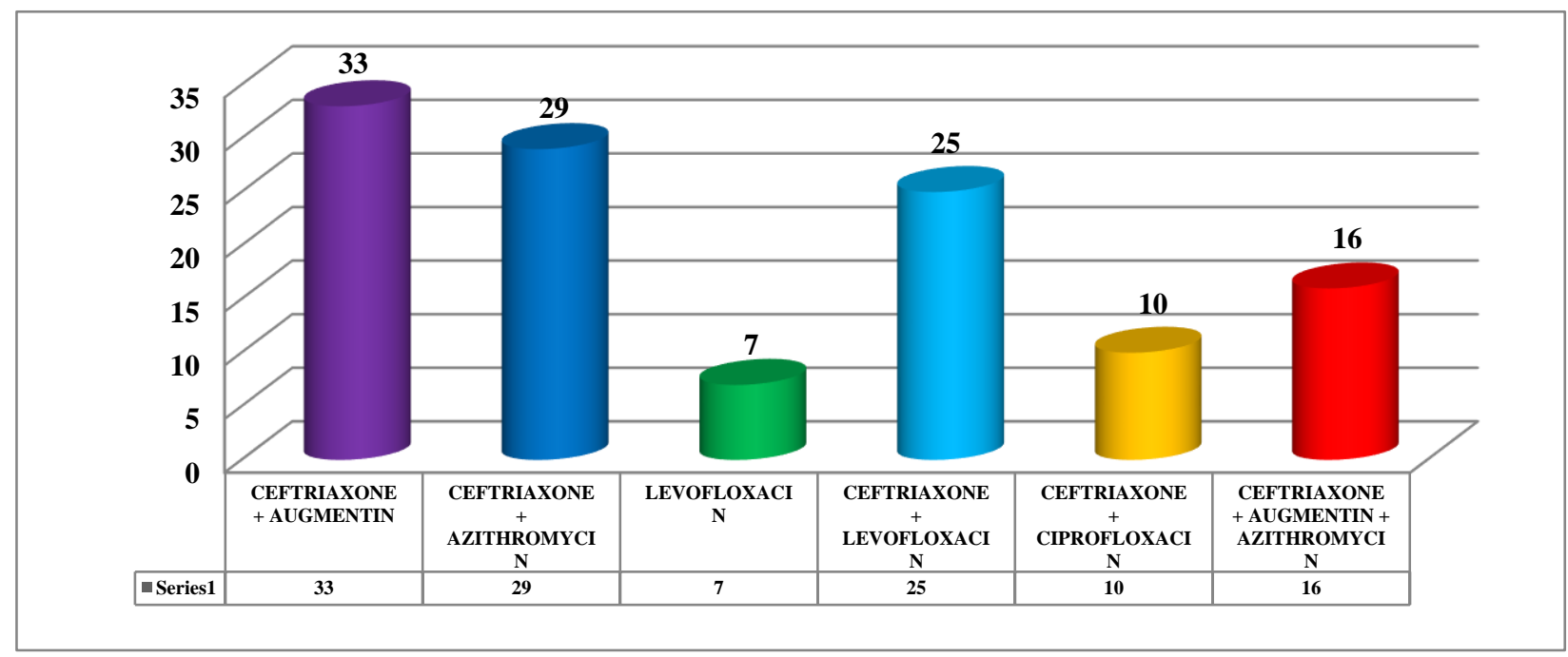

Figure 8: Patients based on antibiotic regimen.

\section{Patient distribution based on antibiotic regimen}

In a total of 120 patients, 33 patients were prescribed with CEF and AUG constituted for 27.5\%, 29 patients were prescribed with CEF and AZI constituted for $24.18 \%, 7$ patients were LEV prescribed with constituted for $5.83 \%$, 25 patients were prescribed with CEF and LEV constituted for $20.83 \%, 10$ patients were prescribed with CEF and CIP constituted for $8.33 \%$ and 16 patients were prescribed with CEF, AUG, AZI constituted for $13.33 \%$.

\section{Patient distribution based on type of empirical therapy}

In a total of 120 patients, 7 patients received mono antibiotic therapy constituted for $87.50 \%, 97$ patients received dual antibiotic regimen constituted for $87.50 \%$ and 16 patients treated with triple antibiotic regimen constituted for $12.50 \%$.

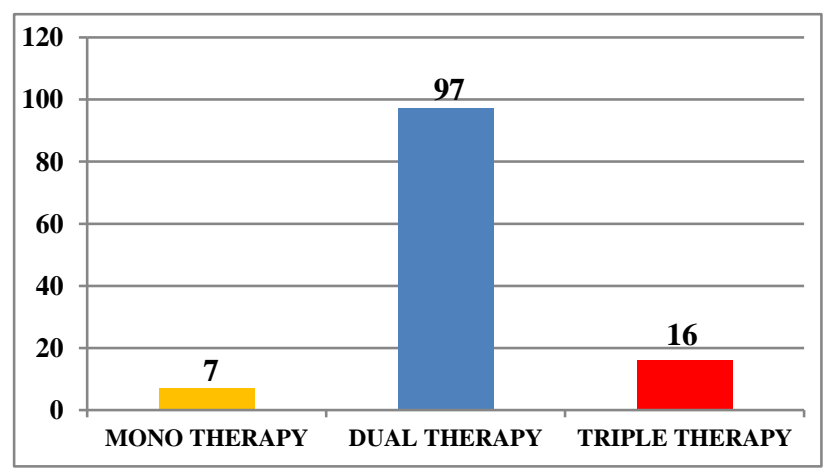

Figure 9: Types of empirical therapy. 


\section{Patient distribution based on length of hospital stay}

It is a time period that exists between date of admission (DOA) into the hospital and date of discharge (DOD) from the hospital. The minimum duration of hospitalization was 3 days and maximum duration was 14 days. Of 120 patients, 55 patients had 3-5 days of hospital stay, 45 patients had 6-8 days of hospital stay, 8 patients had 9-11 days of hospital stay and 12 patients had 12-14 days of hospital stay.

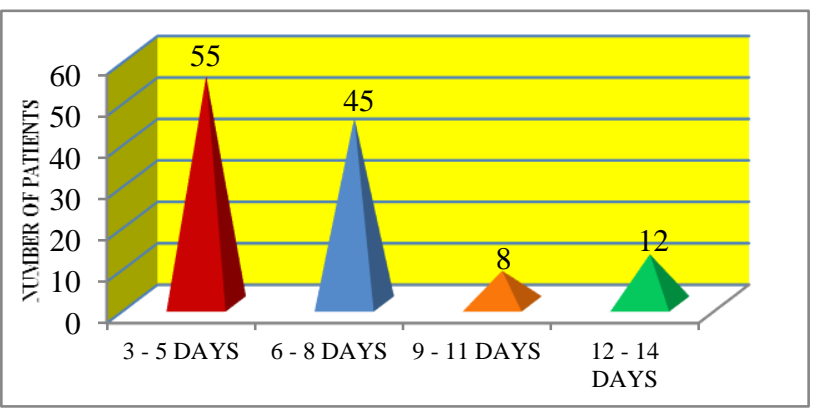

Figure 10: Patients based on duration of hospital stay.

\section{DISCUSSION}

At first, we started with simple parameter i.e., patient's gender. Males $(64.17 \%)$ are being more affected with CAP than females $(36.83 \%)$ and literature like Mustafa et al and Siddalingappa et al has got same results. ${ }^{8,9}$ Coming to the age parameter the patient with age group of 46-55 years are more affected i.e., $31.67 \%$ and 31 patients were in 56 and above years age group constituted for $25.83 \%$ than the patients with other aged group, but literature of Kumar et al has concluded that 65 to 75 years age group patients are more affected with CAP. ${ }^{10}$ The patients with $<18.5$ body mass index are highly affected with CAP. We found that patient with co-morbidities are highly susceptible to CAP than normal individuals and the patients with respiratory tract co-morbidities are high prone to CAP. In a total of 120 patients, 58 patients had co-morbid conditions of which 17 patients had comorbidities with COPD constituted $29.31 \%$, for 6 patients had co-morbidities with asthma constituted for $10.34 \%$, 12 patients had co-morbidities with hypertension constituted for $20.69 \%, 14$ patients had co-morbidities with type 2 diabetes mellitus constituted for $24.14 \%, 4$ patients had co-morbidities with hypothyroidism constituted for $6.90 \%, 2$ patients had co-morbidities with epilepsy constituted for $3.45 \%, 3$ patients had comorbidities with IHD constituted for $5.17 \%$. that is supported by Kumar et al literature. ${ }^{10}$ We also analyzed social habitats of patients, found that habituated patients were more affected with CAP than non-habituated patient and patients with smoking habit have higher incidence rates of occurring CAP.

In our research study, 120 patients who were recruited according to inclusion criteria have undergone radiological examinations such as chest $\mathrm{X}$-ray and $\mathrm{CT}$ chest and found that 105 patients had single-lobe Infiltration constituted for $87.50 \%$ and remaining 15 patients had multi-lobe infiltration constituted for $12.50 \%$, this result is supported by literatures Mustafa et al and Ayesha. ${ }^{8,11} 95$ patients had undergone sputum culture test and remaining 25 patients did not undergo sputum culture tests out of 95 subjects, bacteria was isolated in 68 patients, fungus was isolated in 21 patients and virus was isolated in 6 patients, coincided with Taura et al and Ramana et al. ${ }^{12,13}$ literatures. In a total of 68 samples, it was found that Streptococcus pneumoniae was isolated in 23 patients constituted for $33.82 \%$, Pseudomons aeruginosa was isolated in 19 patients constituted for $27.94 \%$, Klebsiella pneumoniae was isolated in 11 patients constituted for $16.18 \%$, Escherichia coli was isolated in 7 patients constituted for $10.29 \%$, Streptococcus aureus was isolated in 6 patients constituted for $8.82 \%$ and Hemophilus influenzae was isolated in 2 patients constituted for $2.95 \%$ and compared with Taura et al, Ramana et al and Zafar et al literatures got the same results. ${ }^{12-14}$ In a total of 120 patients, 33 patients were prescribed with CEF and AUG constituted for $27.5 \%, 29$ patients were prescribed with CEF and AZI constituted for $24.18 \%, 7$ patients were LEV prescribed with constituted for $5.83 \%, 25$ patients were prescribed with CEF and LEV constituted for 20.83\%, 10 patients were prescribed with CEF and CIF constituted for $8.33 \%$ and 16 patients were prescribed with CEF, AUG and AZI constituted for $13.33 \%$. Literature like Meng Tse et al had connived that $\beta$-lactam alone was the most common antibiotic regimen prescribed for patients and give support to our study. ${ }^{15}$ Literatures of Okesola et al and Li et al gave the evidence that matches to our study. ${ }^{16,17}$

In a total of 120 patients, 7 patients received mono antibiotic therapy constituted for $87.50 \%$. 97 patients received dual antibiotic regimen constituted for $87.50 \%$ and 16 patients treated with triple antibiotic regimen constituted for $12.50 \%$ found the same pattern of results in literature of Meng Tse et al and Taura et al. ${ }^{12,15}$ Length of hospital stay is defined as time period that exists between DOA into the hospital and DOD from the hospital. In our study, minimum duration of hospitalization for CAP patients was 3 days and maximum duration was 14 days. In a total of 120 patients, 55 patients had 3 to 5 days of hospital stay, 45 patients had 6 to 8 days of hospital stay, 8 patients had 9 to 11 days of hospital stay and 12 patients had 12 to 14 days of hospital stay and same conclusion was given Mustafa et al, Kumar et al literature. ${ }^{8,10}$ This clearly indicates that the symptoms will be free with in less time in case of pneumonia.

\section{CONCLUSION}

This research work clearly highlights the study of empirical treatment of antibiotic drug regimens in patients with CAP in a tertiary care teaching hospital. Male patients had higher incidence rates of occurring CAP when compared to female patients. Maximum number of 
patients who were diagnosed with CAP belongs to 45-55 years and above age group. Patients with social habits like smoking, alcoholism, betel nut chewing have increased risk of occurring CAP when compared to patients without social habits. The risk of developing CAP was high in patients with co-morbidities like COPD, asthma, type 2 diabetes mellitus and hypertension. Empirical treatment with $\beta$-lactam antibiotics was the most commonly prescribed class to CAP patients, in addition, CEF and AUG regimen was highly prescribed. Streptococcus pneumoniae and Pseudomonas aeruginosa were the most common causative micro-organisms for CAP. In patients with atypical pneumonia, triple antibiotic regimen was preferred over dual therapy; also have higher duration of hospitalizations.

\section{ACKNOWLEDGEMENTS}

We wish to express our gratitude to a number of people who made important contributions to the accomplishment of this research article. It is pleasure to convey our gratitude to Dr. Shaik Kareemulla sir, working as Assistant Professor in P. Ramireddy college of pharmacy for his supervision, advice and guidance from the early stage of our work as well as his extraordinary support, encouragement throughout the work. We would also like to extend appreciations to individuals who involved in data collection and the study participant who was willingly gave us all the information we needed without any reservation.

Funding: No funding sources

Conflict of interest: None declared

Ethical approval: The study was approved by the Institutional Ethics Committee

\section{REFERENCES}

1. Walker R, Whittlesea C. Clinical pharmacy and therapeutics. 5th edition. Churchill Livingstone Elsevier; 2014: 550-552.

2. Niederman MS, Craven DE, Bonten MJ, et al. American Thoracic Society, Infectious Diseases Society of America. Guidelines for the management of adults with hospital-acquired (HAP), ventilatorassociated (VAP) and healthcare-associated pneumonia; Am J Res Cri Care Med. 2005;17(1);388-416.

3. Jain S, Williams DJ. Community acquired pneumonia requiring hospitalization among U.S. adults. N Engl J Med. 2015;373(5):415-27.

4. Walker B, Colledge NR, Ralston S, Penman I. Davidson's principles and practice of medicine. 22nd edition. Churchill Livingstone, Elsevier; 2014: 682-685.

5. Dev Y. Pathophysiology of community acquired pneumonia. JAPE. 2012;60(1):8-9.

6. Moore M, Stuart B, Little P, Smith S, Thompson MJ, Knox K, et al. Diagnosis of pneumonia in lower respiratory tract infections;3c prospective cough complication cohort study. Europe Respire J. 2017;50(3):151-3.

7. Dan LL, Antbony FS, Dennis LK, Stephen LH, Larry JJ. Harrison's principle of internal medicine, 18thedition. Volume 2. The McGraw-Hill companies; 2011: 2134-5.

8. Saeed MS, Aldakheel BA. Prescribing Patterns of Antibiotics for Community-Acquired Pneumonia in Adult in King Saud Hospital. British J Med Medical Res. 2017;20(5):1-8.

9. Siddalingappa CM, Kalpana L, Puli S, Vasudha TK, Acharya A. Sensitivity pattern of bacteria causing respiratory tract infections in a tertiary care centre. Int J Basic Clin Pharmacol. 2013;2:590-5.

10. Kumar S, Agrawal D, Santra S, Dehury S, Das P, Swain T. Prescribing pattern of antibiotics in community-acquired pneumonia in a teaching hospital of Southeast Asia; J Health Res Rev. 2015;2(3):86.

11. Fatima A, Naqvi SB, Khaliq SA, Perveen S, Jabeen S. Antimicrobial susceptibility pattern of clinical isolates of Pseudomonas aeruginosa isolated from patients of lower respiratory tract infections. Springer Plus. 2012;70:12-24.

12. Taura DW, Hassan A, Yayo AM, Takalmawa H. Bacterial isolates of the respiratory tract infection and their current sensitivity pattern among patients attending Aminu Kano Teaching Hospital KanoNigeria. Int Res J Microbiol. 2013;4(9):226-31.

13. Ramana KV, Kalaskar A, Rao M, Rao SD. Aetiology and Antimicrobial Susceptibility Patterns of Lower Respiratory Tract Infections (LRTI's) in a Rural Tertiary Care Teaching Hospital at Karimnagar, South India. Am J Infec Dis Microbiol. 2013;1(5):101-5.

14. Zafar A, Hussain Z, Lomama E, Sibiie S, Irfan S, Khan E. Antibiotic susceptibility of pathogens isolated from patients with community-acquired respiratory tract infections in Pakistan-the active study. J Ayub Med Coll Abbottabad. 2008;20(1):7-9.

15. Meng TSE, Lee SH, Chang SS, Chan YL, Pang L, Hsu SM, et al. Comparative treatment failure rates of respiratory fluoroquinolones or $\beta$-Lactam alone in the treatment for community acquired pneumonia in adult outpatients. Medicine. 2015;94(39):1662.

16. Okesola AO, Ige OM. Trends in Bacterial Pathogens of Lower Respiratory Tract Infections. Indian J Chest Dis Allied Sci. 2008;50:269-72.

17. Li JZ, Winston LG, Moore DH. Bent Efficacy of short-course antibiotic regimens for communityacquired pneumonia; A meta-analysis. Am J Medicine. 2007;120(9):783-90.

Cite this article as: Leela Prasad Babu K, Kareemulla S, Sai Pravalika SK, Nishad Khan P, Venkateswarlu G, Sreeharinadh K. Antibiotic regimens utilization in treating community acquired pneumonia of a government practice setting: a prospective observational study in medical inpatients. Int J Basic Clin Pharmacol 2020;9:336-41. 